\title{
The Stability of Supported Liquid Membranes
}

\author{
A.M. NEPLENBROEK, D. BARGEMAN and C.A. SMOLDERS \\ University of Twente, Department of Chemical Engineering, P.O.B. 217, 7500 AE Enschede \\ (The Netherlands)
}

(Received February 24, 1991)

\section{SUMMARY}

In this paper a new hypothesis about the instability mechanism of SLMs will be discussed: emulsion formation induced by lateral shear forces. Experimental results show that a water phase with a low salt concentration which flows along the membrane interface causes the removal of both solvent and carrier from the membrane. There is a significant correlation between the instability of the liquid membrane and the stability of emulsions formed with the same system. Therefore, the development of stable SLMs needs conditions in which formation of relatively stable emulsions is prevented. This can be realized by gelation of the liquid membrane. A gel network was created in the pores of the membrane in such a way that the permeability is not decreased while the stability increases to values which are very promising.

\section{INTRODUCTION}

The use of supported liquid membranes (SLMs) in which an extraction liquid containing a carrier is immobilized in the pores of a microporous support has recently been introduced as a promising new separation technique. Some advantages ascribed to this method are the high selectivity, the possibility to concentrate ions by coupled transport and the high diffusion rate through the liquid. Despite these advantages SLMs are, until now, not widely used for practical separation processes. One of the main reasons for this is the instability of the SLMs, which is caused by the removal of the membrane liquid from the pores of the support. The consequences of this removal are that the permeability of the membrane is reduced or that the membrane starts to leak. 
It is obvious that the solubility of components from the LM phase into the aqueous phases may cause SLM-instability effects. It is also commonplace that the LM phase is forced out of the pores of the support because of a pressure difference over the membrane which exceeds a critical value. However, in practice it appears that even by preventing LM-components to dissolve into the aqueous phase and by an absence of a pressure difference over the membrane, instability effects do occur. Danesi et al. [1] and Fabiani et al. [2] explain these effects by an osmotic mechanism for transport of water. In this model the LM phase is supposed to be removed from the pores by a flow of water through the membrane as a consequence of the combined effect of the solubility of water in the LM phase and an osmoticpressure gradient over the membrane.

We seriously doubt that the solubility of only a few percent of water in the organic phase can result in a convective water flow because there is no continuous water path present for the aqueous phase in the membrane, necessary for the osmotic forces to be effective. Therefore, another mechanism must be responsible for the removal of the LM phase from the pores. In this article results of research efforts concerning the factors which influence the stability of SLMs will be described to get more insight in the mechanism of SLM-degradation. Furthermore, a new technique to stabilize SLMs will be shown.

An SLM system, in which nitrate ions are removed from water by a counter transport of chloride ions, is used as a model system (with possibility for practical application). For these measurements quaternary ammonium salts were used as carriers.

\section{LONG-TERM PERMEATION}

The course of the flux as a function of time is an important aspect in SLM stability. It is possible that this behaviour is determined especially by the nature of the carrier used. Therefore, long-term permeability is determined for membranes with different carriers: tetraoctylammonium (TeOA) and trioctylmethylammonium (TOMA) salt $(0.2 \mathrm{M})$. As solvent o-nitrophenyloctylether (o-NPOE) was used and as support Celgard ${ }^{\oplus} 2500$. The aqueous phases which flowed parallel to the membrane day and night had a composition of $0.004 \mathrm{M} \mathrm{NaNO}_{3}$ (feed) and $4.0 \mathrm{M} \mathrm{NaCl}$ (stripping phase). The nitrate flux was calculated from the slope of the curve for the nitrate concentration as a function of time. After 3 or $4 \mathrm{~d}$ both aqueous solutions were replaced by fresh starting solutions, after which the flux was determined again. In Fig. 1 the long-term permeability behaviour of these two SLMs is given as a function of time. 


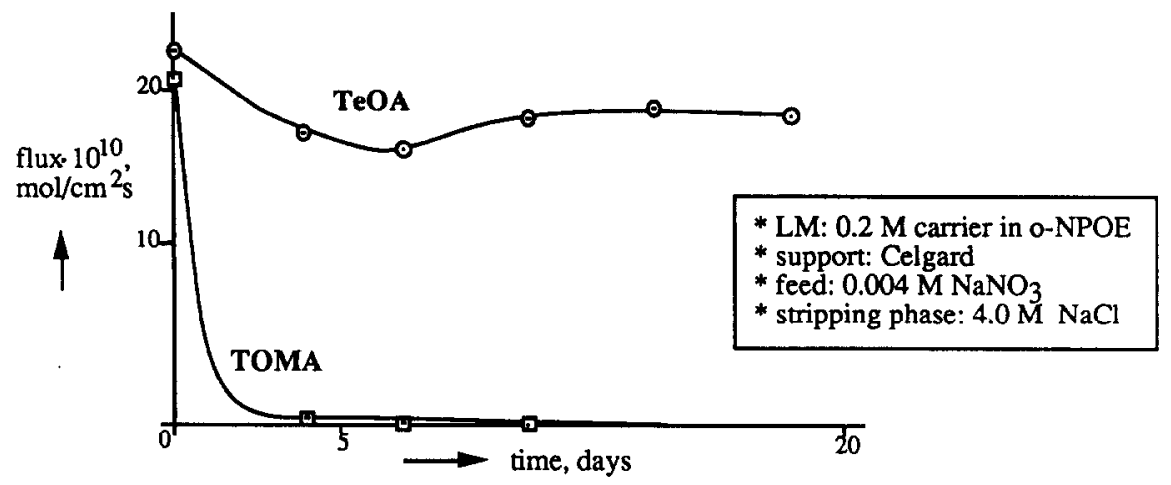

Fig. 1. Long-term permeation; flux as a function of time and type of carrier.

The results given in this figure show that the membrane with TeOA as a carrier gives a nearly constant flux. This means that we are dealing with a reasonably stable membrane. The slight decrease in flux in the beginning is probably caused by the adsorption of air bubbles, which were present in the system, onto the membrane surface. Contrary to this, the membrane with TOMA as a carrier shows an entirely different stability behaviour. Only during the first day the flux is comparable to the case where the TeOA membrane is used, but then it decreases drastically till after about $3 \mathrm{~d}$ the process of nitrate removal has completely stopped. It is obvious that this is due to removal of components of the LM phase, and especially the carrier TOMA, from the membrane.

\section{REMOVAL OF CARRIER}

From the long-term permeation experiments it appeared that the carrier TOMA is removed from the membrane to a much larger extent than TeOA. When shaking aqueous solutions with LM phases in a test tube, in some cases relatively stable emulsions are formed, depending on the composition of the phases. It was found among other things that with TOMA present in the LM phase much more and more stable emulsions were formed than in the presence of TeOA. Based on these parallels the presumption arises that formation of emulsions could be the reason for SLM instability effects. We also found that by increasing the salt concentration in the aqueous phases less stable emulsions were formed. To test the hypothesis that emulsion formation causes SLM instability, the influence of the composition of aqueous solutions on SLM stability has been determined. For this purpose two aqueous solutions with the same composition were streamed parallel to 
the membrane under defined conditions. The amount of carriers TeOA and TOMA removed from the membrane with o-NPOE as solvent was determined spectrophotometrically. Fig. 2 gives the influence of $\mathrm{NaCl}$ concentration in the aqueous phases on the removal of carrier.

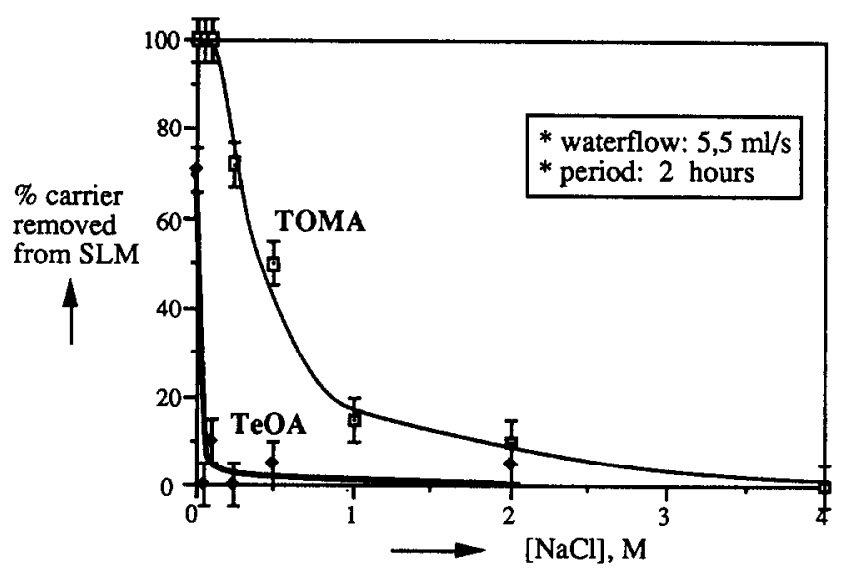

Fig. 2. Carrier removal as a function of $\mathrm{NaCl}$ concentration in the aqueous phase for two different carriers; solvent: o-NPOE; support Celgard; equal salt concentrations in feed and stripping phase.

This figure shows that an increase in $\mathrm{NaCl}$ concentration in the aqueous phases leads to a decrease in the amount of carrier that is removed from the membrane. It also clearly shows that the carrier TOMA is removed to a much larger extent from the membrane than TeOA. This result is in agreement with the result of the long-term permeability experiments, where it was found that a membrane with $\mathrm{TeOA}$ as carrier provides a much more stable membrane. The same behaviour is found for salts like sodium nitrate. We can conclude that in those permeability experiments with different compositions of the aqueous phases the carrier is removed from the membrane at the feed side having a low salt content. However, these results also show that the low salt content of $0.004 \mathrm{M}$ sodium nitrate in the feed during the permeation experiments is already enough to prevent loss of the carrier TeOA from the membrane. This is in agreement with the relatively constant flux of the membrane with $\mathrm{TeOA}$ as carrier as is illustrated in Fig. 1. These results clearly show that an osmotic pressure difference is not necessary to remove the LM phase from the membrane since the compositions of the feed and the stripping phases are equal. Neither could the results be explained by the solubility of LM-components in the aqueous phases. 


\section{EMULSION FORMATION AND STABILITY}

Shaking an LM phase with an aqueous solution leads to a more or less turbid water phase, depending on the composition of the phases. Emulsion formation and stability of o-NPOE solutions with TOMA or TeOA is studied by (hand) shaking an amount of organic phase with an aqueous phase with various concentrations of $\mathrm{NaCl}$ and studying these aqueous phases in a spectrophotometer. The light transmission after $5 \mathrm{~min}$ is given in Fig. 3.

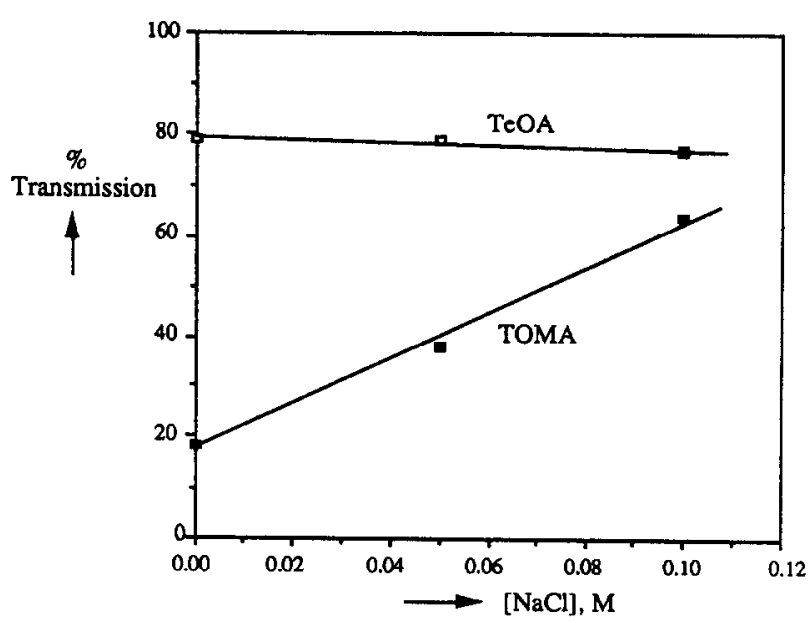

Fig. 3. Light transmission of aqueous phases containing emulsified LM phases as a function of $\mathrm{NaCl}$ concentration and type of carrier; solvent: o-NPOE.

We can conclude from these results that the LM phase with TOMA as carrier is emulsified to a much larger extent than the LM phase with TeOA. This behaviour is caused by the differences in geometric structure of the carriers. We also see in Fig. 3 that with TOMA as surfactant less stable emulsions are formed when the sodium chloride concentration in the water phases is increased. This result can be explained by theories on the stability of charged colloidal systems.

When we combine these results with those of the experiments in which aqueous phases flowed along the membranes, we can conclude that there is a significant correlation between the instability of the membrane and the stability of emulsions prepared from the same system. The membrane is more stable when less stable emulsions are formed between the LM phase and the aqueous phase. Many other results which confirm this hypothesis were obtained. 


\section{SLM DEGRADATION BY EMULSION FORMATION}

In view of this correlation the conclusion is justified that instability of SLMs is induced by the formation of emulsions. A possible mechanism to describe these instability phenomena is that local deformations of the meniscus in the pores of the support lead to the splitting off of emulsion droplets. Such local deformations are given schematically in Fig. 4 . These deformations (also called "Kelvin-Helmholz instabilities") arise when two phases move with different velocities parallel to the interface. It causes, for instance, waves to develop on a water surface over which the wind blows. A possible cause for the splitting off of emulsions when the meniscus of the LM phase in the pore of the support is locally deformed is the formation of interfacial tension gradients which result in Marangoni effects.

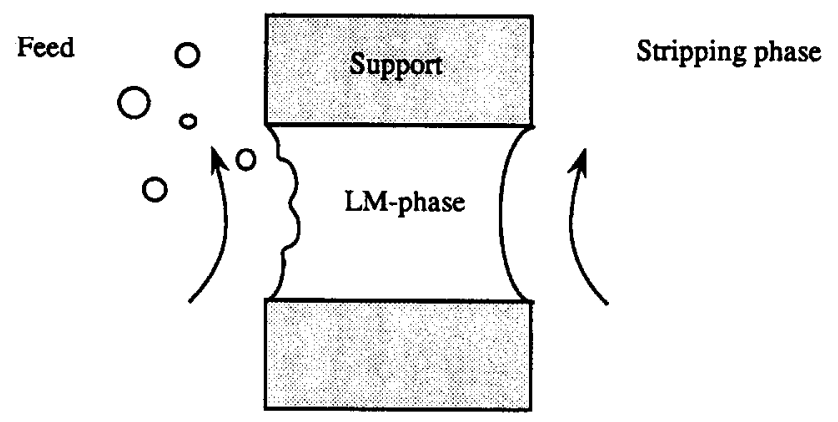

Fig. 4. SLM degradation by emulsion formation due to local deformation of LM phase in the pores of the support.

However, it is clear that for the development of stable SLMs the composition of the LM phase has to be chosen in such a way that it has little capacity for the formation of emulsions. Especially the structure of the carrier is important. Furthermore, the instability effects can be diminished by varying the composition of the water phases. Of course, the boundary conditions are determined by the total process of the SLM system. The membranes can also be stabilized when the deformation of the meniscus of the LM phase in the support pores is prevented. A technique to realize this is to gel the LM phase.

\section{SLM STABILIZATION BY GELATION}

Two different kinds of gelled SLMs were made. In Fig. 5 the effect of a homogeneous gel network on the fixation of the LM phase in the pores of 
a support is presented schematically. By gelling the membrane liquid its overall viscosity will increase, and thus the resistance against liquid displacement out of the membrane pores will be enhanced. Therefore, the formation of emulsion droplets will be prevented, and the stability of the membrane will increase.

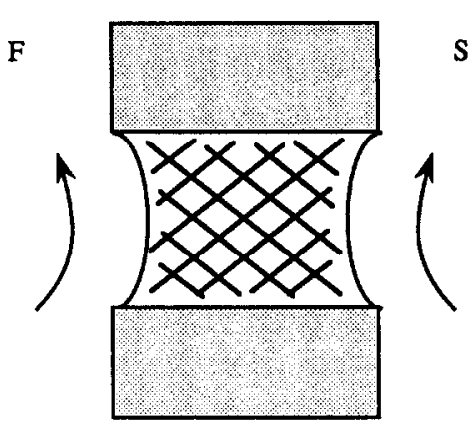

Fig. 5. SLM stabilization by gelation: homogeneous gel network in the LM phase.

Results of long-term permeability measurements performed in the same way as described in Fig. 1 show that the presence of a gel network leads to a substantial increase in membrane stability. A number of experiments were performed in which the membranes contained 5\% PVC. In all cases the stability of the membrane increased by a factor of 2-5 to at least 4 months. Stability means, in this context, that no direct contact between the feed and stripping phase occurs, which leads to non-facilitated transport. At the same time the permeability decreases only to a small degree because of the open gel structure.

The second type of gelled membrane is given schematically in Fig. 6 . These membranes contained only one dense gel layer because in this way the formation of emulsion droplets at the interface with the feed can be prevented as specifically as possible.

Relatively high polymer concentrations can be used without reducing the flux because these gel layers are very thin in relation to the total thickness of the membrane. It appeared that in all cases the gel layers were thinner than $2 \mu \mathrm{m}$, while the total membrane thickness was $100 \mu \mathrm{m}$. The gel layers that were applied contained $40 \%$ polymer and $60 \%$ LM-phase.

Also experiments were performed in which a gel network was created with a shorter mesh size. This was realized by cross-linking the polymer not only physically but also chemically. For this purpose PVC with carboxylic acid groups was used as the starting material and a diamine as the cross- 
linking agent. An activator is needed to perform the chemical cross-linking reaction at room temperature. DCC (dicyclohexylcarbodi-imide) was used as an activator. Based on previous results, it could be expected that longterm permeability measurements under normal conditions (salt content in the feed: $0.004 \mathrm{M} \mathrm{NaNO}_{3}$ ) would require a very long test time, since for an SLM without a gel layer a stability of 13 weeks was found under the conditions of concentrating experiments. Therefore, it was decided to investigate the influence of the gel layers under conditions where the stability of the SLM was diminished. This is realized by flowing an aqueous phase with a very low salt content $\left(10^{-4} \mathrm{M} \mathrm{NaCl}\right)$ along the feed side of the membrane instead of the normal feed solution with a sodium nitrate concentration of $0.004 \mathrm{M}$. This means that the loss of carrier is enlarged, leading to much smaller experimental measuring times. Therefore, the degradation process of the membrane is accelerated on purpose.

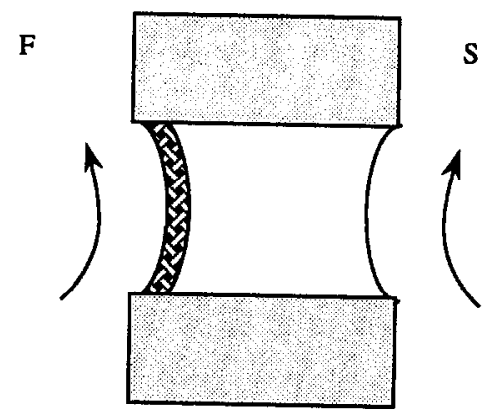

Fig. 6. SLM stabilization by gelation: one dense gel layer at the interface with the feed.

The stability of the membrane is determined by measuring the permeability of the membranes in the usual way with a feed solution of $4 \cdot 10^{-3} \mathrm{M}$ $\mathrm{NaNO}_{3}$ and a stripping phase of $4 \mathrm{M} \mathrm{NaCl}$. After about $5 \mathrm{~h}$ the "degradation process conditions" were installed by replacing the feed solution by the aqueous solution of $10^{-4} \mathrm{NaCl}$ which then was passed along the membrane. The next day the stripping phase was replaced by a fresh solution of $4 \mathrm{M}$ $\mathrm{NaCl}$ and the feed by a $4 \cdot 10^{-3} \mathrm{M} \mathrm{NaNO}_{3}$ solution, and the flux was again determined. The degradation process conditions then were reestablished after the flux measurement. This sequence of actions was repeated every $24 \mathrm{~h}$.

In Fig. 7 the influence of those gel layers on the permeability is given. The flux is plotted as a function of the "degradation" time, that is the time that the membrane was in contact with the water phase with the very low salt 
content at the feed side. This means that it was assumed that the flux value is not diminished during the time needed to measure the flux, since these measurements were performed with a higher salt content. Curve 1 is for a membrane without a gel layer, while the other membranes do have a gel layer. Curve 2 is for a membrane with carboxylated PVC, but without crosslinkers or activator. Curve 3 is for a membrane with a gel layer with only the activator DCC and without a diamine. Curves 4 and 5 are for membranes with different amounts of activator and different types of cross-linkers. In the case of Curve 4 dodecanediamine was used and for Curve 5 phenylenediamine was used as the cross-linking agent.

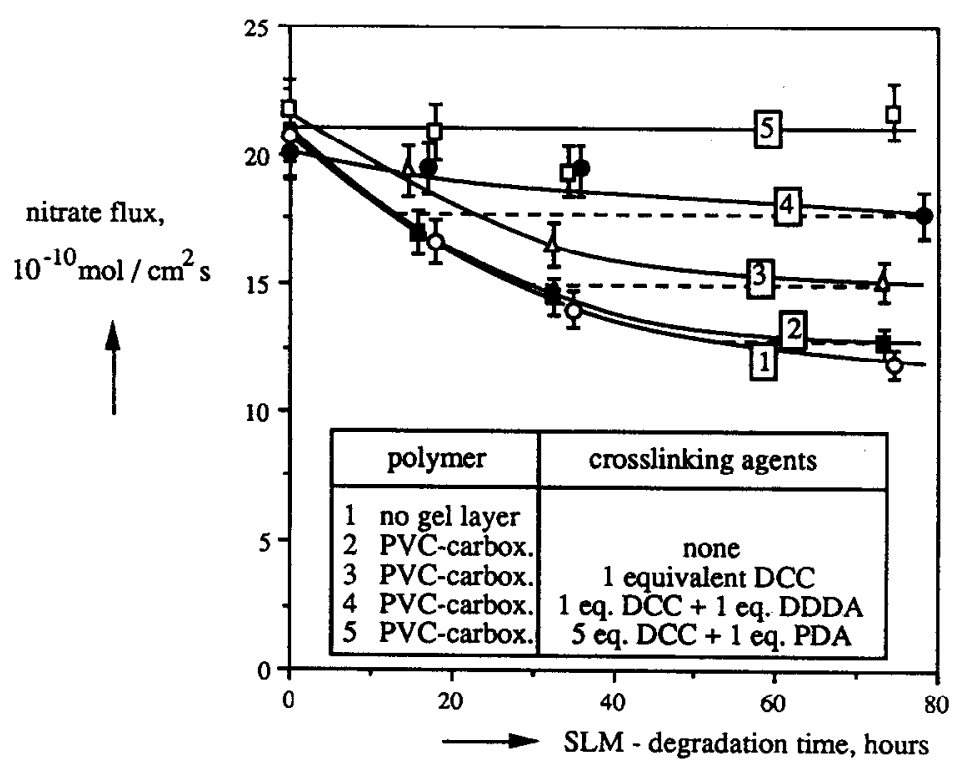

Fig. 7. Influence of the presence of a gel network on nitrate flux; solvent o-NPOE; carrier TeOA; polymer concentration in gel layer $40 \%$; thickness of gel layer $<2 \mu \mathrm{m}$; support Accurel; feed solution: alternating $10^{-4} \mathrm{NaCl} / 4 \cdot 10^{-3} \mathrm{M} \mathrm{NaNO}_{3}$ stripping phase $4 \mathrm{M} \mathrm{NaCl}$; water flow rate $5.5 \mathrm{ml} / \mathrm{s}$.

A remarkable result is obtained when the initial flux values of the different curves are compared. It appears that the presence of the gel network has no measurable influence on the permeability of the membrane. This is explained by the fact that the thickness of the applied gel layer is very thin in relation to the total thickness of the membrane.

Fig. 7 shows that the flux of Curve 1, without a gel layer, decreases considerably in a period of $80 \mathrm{~h}$ under the conditions given. On the contrary, 
this flux reduction is smaller for the membranes with a gel network. From these results the real long-term permeabilities can be calculated because the stability of a membrane without a gel network is known. In other long-term permeation measurements it has been determined that membrane 1 has a stability of 13 weeks under the conditions of concentrating experiments; that means a high feed volume of more than $4 \mathrm{l}$ and aqueous phases that were refreshed every week. The real long-term permeability of the membranes with a dense gel layer can be calculated by comparing the observed "simulated" long-term permeability with that of a membrane without a gel network. By doing this a predicted long-term stability of membrane 3 of about 40 weeks is obtained; membrane 4 has a stability of 80 weeks, while membrane 5 will be stable for some years.

\section{CONCLUSIONS}

The results showed that the instability of SLMs is caused by a loss of the membrane phase through the formation of emulsion droplets. A technique to suppress this degradation of the membrane is to apply a thin gel layer at the feed side of the LM phase. This treatment has no negative influence on the flux because the thickness of the dense gel layer is very small. From the results it can be concluded that the most stable membranes are formed by a gel network with a short mesh size which is built with chemically stable cross-links. It appears that especially these types of membranes have predicted stabilities which seem very interesting for practical applications. Therefore, it can be concluded that these asymmetrically gelled membranes open new perspectives for practical application.

\section{REFERENCES}

1 P.R. Danesi, L. Reichley-Yinger and P.G. Rickert, Life-time of supported liquid membranes: the influence of interfacial properties, chemical composition and water transport on the longterm stability of the membranes, J. Membr. Sci., 31 (1987) 117-145.

2 C. Fabiani, M. Merigiola, G. Scibona and A.M. Castagnola, Degradation of supported liquid membranes under an osmotic pressure gradient, J. Membr. Sci., 30 (1987) 97-104.

3 A.M. Neplenbroek, Stability of Supported Liquid Membranes, Dissertation, University of Twente, 1989. 